\title{
Spatial Training and High-Frequency Stimulation Engage a Common Pathway to Enhance Glutamate Release in the Hippocampus
}

\author{
Gal Richter-Levin, ${ }^{1}$ Laura Canevari, and Tim V.P. Bliss \\ Division of Neurophysiology and Neuropharmacology \\ National Institute for Medical Research (NIMR) \\ London NW7 1AA, UK
}

\begin{abstract}
We have measured depolarizationinduced release of endogenous glutamate in synaptosomes prepared from the dentate gyrus after the induction of LTP by high-frequency stimulation in anesthetized rats, and after training in the water maze. Both spatial training and LTP in untrained rats were accompanied by an increase in glutamate release from dentate synaptosomes. The enhancement of synaptosomal glutamate release induced by high-frequency stimulation was abolished in well-trained rats, and was reduced in partially trained rats and in rats trained in a nonspatial task. However, the magnitude of LTP was similar in well-trained and untrained groups. These results indicate that spatial training activates a glutamate release pathway that converges with that activated in LTP, and demonstrate an unexpected dissociation between increased glutamate release and LTP.
\end{abstract}

\section{Introduction}

Long-term potentiation (LTP) in the hippocampus has been studied extensively as a potential synaptic mechanism for learning, and a large body of correlational evidence has been adduced to support the hypothesis that LTP and learning are causally linked (Morris et al. 1986; Grant et al. 1992;

\footnotetext{
${ }^{1}$ Corresponding author.

Present address: Department of Psychology, University of Haifa, Mount Carmel, Haifa 31905 Israel.
}

Silva et al. 1992; Barnes 1995; but see Jeffery 1997). An example of this approach is to look for biochemical correlates of LTP and then establish whether similar changes occur with learning. In the dentate gyrus, a subfield of the hippocampal formation that is required for spatial learning (McNaughton et al. 1989), LTP is associated with enhanced glutamate efflux in vivo (Dolphin et al. 1982; Bliss et al. 1986; Errington et al. 1987) and ex vivo (Bliss et al. 1987; Canevari et al. 1994). A similar effect is seen in area CA1 (Ghisjen et al. 1992). There is evidence that learning is associated with a similar increase in glutamate efflux in the dentate gyrus. Laroche and his colleagues found an NMDA receptor-dependent increase in depolarization-induced release of radiolabeled glutamate in slices from the dentate gyru of conditioned as compared to pseudoconditioned animals in a food-motivated lever-pressing task (Laroche et al. 1987; Redini-Del Negro and Laroche 1993), and more recently, increases in both endogenous (Richter-Levin et al. 1995) and radiolabeled (McGahon et al. 1996) glutamate efflux have been reported following training in a spatial learning task, the Morris water maze.

In the present experiments we have asked whether the increased glutamate release seen in LTP and in spatial learning is mediated by a common biochemical pathway. After training in a spatial or nonspatial version of the water maze, rats were anesthetized and high-frequency stimulation was applied unilaterally to the perforant path to induce LTP. Two hours after the tetanus, synaptosomes were prepared and stimulation of glutamate release by veratradine was induced. Using this protocol, we compared glutamate release in the potentiated and nonpotentiated sides of the same animal, for rats in both trained and non-trained

LEARNING \& MEMORY 4:445-450 @ 1997 by Cold Spring Harbor Laboratory Press ISSN1072-0502/97 \$5.00

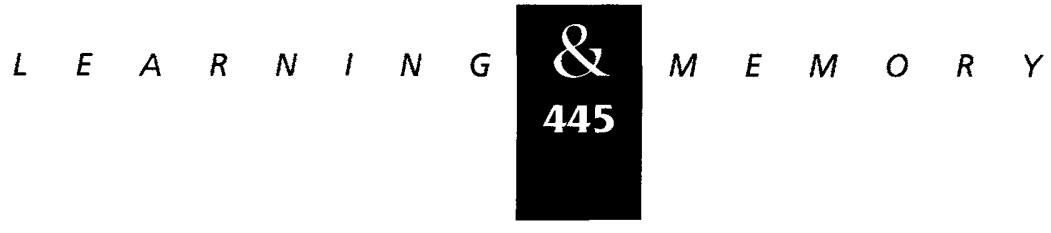


Richter-Levin et al.

groups. In this way we were able to bring the statistical power of within-animal comparisons to bear on the differences between the groups. In trained animals, there was no further increase in glutamate release following high-frequency stimulation, indicating that similar mechanisms are involved in the enhancement of depolarization-induced glutamate release following a tetanus and in spatial training. However, the fact that the magnitude of LTP was similar in both trained and untrained animals complicates any straightforward interpretation of our results as providing evidence for the hypothesis that LTP is a necessary neural substrate of spatial learning.

\section{Materials and Methods}

\section{ANIMALS}

Subjects were male Sprague-Dawley rats (200-350 grams, bred at NIMR), randomly divided into five groups: Naive $(n=6), 1$ Block $(n=5), 2$ Blocks $(n=5), 3$ Blocks $(n=6)$, and Visible $(n=5)$, according to the type and degree of training, as described below.

\section{BEHAVIORAL PROCEDURES}

Rats in the 1,2, and 3 Blocks groups were trained in the Morris water maze to locate a submerged platform (diam. $12 \mathrm{~cm}$ ) in a pool (diam. 1.5 m) filled with opaque water at room temperature (Morris 1984). Trials were given in blocks of six, with 5 min between trials. Rats were released from one of three different starting blocks in semirandom order and the escape latency measured. The second block in the 2 Blocks and 3 Blocks groups was given $6 \mathrm{hr}$ after the first, and the third block in the 3 Blocks group 5 days later. For the Visible group the platform was placed $2 \mathrm{~cm}$ above the water level and its position was altered randomly after each trial. The Naive group was not exposed to the water maze.

\section{ELECTROPHYSIOLOGY}

One hour after the last trial rats were anesthetized (urethane, $1.2 \mathrm{~g} / \mathrm{kg}$, i.p.) and prepared for stimulation and recording (Richter-Levin et al. 1994). Briefly, anesthetized rats were placed in a head-holder and holes drilled in the skull to allow the insertion of a bipolar stimulating electrode (two insulated and twisted nichrome wires, each $125 \mu \mathrm{m}$ diam., Goodfellow) into the angular bundle (coordinates: $4.4 \mathrm{~mm}$ lateral, $8.0 \mathrm{~mm}$ posterior to bregma), and a recording electrode (insulated nichrome wire, $125 \mu \mathrm{m}$ diam.) into the hilar region of the dentate gyrus (coordinates: $2.5 \mathrm{~mm}$ lateral, $4.0 \mathrm{~mm}$ posterior to bregma). The positions of the stimulating and recording electrodes were adjusted to maximize the slope of the positive-going field EPSP. Test stimuli (monopolar pulses, 50$\mu$ sec duration, intensity adjusted to yield a population spike that was $20 \%-40 \%$ of maximal pretetanus value) were delivered at $0.033 \mathrm{~Hz}$ throughout the experiment. After the field potential had stabilized, baseline responses were collected for 10 min, followed by a tetanus designed to produce maximal LTP (six trains of eight pulses at $400 \mathrm{~Hz}$, intertrain interval $4 \mathrm{~min}$, pulse duration $150 \mu \mathrm{sec}$, same intensity as test stimulus). Potentials were recorded for $1 \mathrm{hr}$ after the tetanus symptosome. Responses were filtered (3-dB points at 1 and 3 $\mathrm{kHz}$ ), digitized at $10 \mathrm{kHz}$, and stored to disk for offline analysis. The magnitude of LTP was calculated from the mean value of the slope of the EPSP for 10 responses at the end of the first hour after the tetanus, expressed as a percentage of the mean value during the baseline recording period. Stimulus timing, data collection, and analysis were controlled by computer. During recording, rats were maintained at $37^{\circ} \mathrm{C}$ with a homeothermic blanket system (Harvard).

\section{ASSESSMENT OF GLUTAMATE RELEASE}

Rats were decapitated $2 \mathrm{hr}$ after the tetanus, the brains removed, and the dentate gyri taken for preparation of synaptosomes (Whittaker 1969; Lynch and Voss 1990). Samples from each rat were incubated with veratridine (Veratrine, Sigma; 50 $\mu \mathrm{M})$ for $15 \mathrm{~min}$ at $30^{\circ} \mathrm{C}$. The particulate and extrasynaptosomal fractions were separated and the latter subjected to amino acid analysis by HPLC and fluorometry (Canevari et al. 1992). Note that our experimental design called for LTP to be induced within $2 \mathrm{hr}$ of the final trial in the water maze, followed immediately by the preparation of synaptosomes and stimulation of glutamate release. It was not possible to do more than one, or at most two, such experiments per day. The results presented here (see Table 1) were thus collected over a period of many weeks. In analyzing the data we have concentrated on the differences in release 
Table 1: Absolute values of glutamate release and the percent difference between the two sides

\begin{tabular}{lccrrr}
\hline & \multicolumn{1}{c}{ Naive } & 1 Block & 2 Blocks & \multicolumn{1}{c}{ 3 Blocks } & Visible \\
\hline Nonpotentiated side & $14.8 \pm 0.9$ & $17.4 \pm 1.6$ & $25.0 \pm 3.2$ & $22.9 \pm 2.4$ & $22.4 \pm 1.2$ \\
Potentiated side & $17.4 \pm 1.1$ & $18.6 \pm 1.5$ & $26.0 \pm 3.2$ & $23.4 \pm 2.8$ & $24.3 \pm 1.6$ \\
Percent difference & $19.2 \pm 2.3^{* *}$ & $7.3 \pm 1.8^{*}$ & $5.2 \pm 5.9$ & $3.2 \pm 3.8$ & $8.1 \pm 2.3^{*}$ \\
\hline
\end{tabular}

Glutamate release nmoles $/ \mathrm{ml} / \mathrm{mg}$ protein. Percent difference; $\left(^{*}\right) P<0.05 ;\left(^{* *}\right) P<0.01$.

between potentiated and nonpotentiated sides of each animal, which were always analyzed in the same HPLC run, rather than the absolute values in different animals, which may have been tested several weeks apart.

\section{STATISTICAL ANALYSIS}

For between-group comparisons one-way ANOVA was employed, followed by Student-Newmann-Keuls post hoc tests between pairs of groups; paired, two-tailed $t$-tests were used for within-animal comparisons of potentiated and nonpotentiated sides. Results are presented as means \pm S.E.M.

\section{Results}

During the first block of six trials in the water maze a significant improvement in performance was observed in all three groups (Fig. 1A), but there was no obvious spatial learning (the rats changed their behavior from trying to escape from the pool to exploring it, but displayed no clear orientation toward the actual location of the hidden platform). By the end of the second block in the 2 and 3 Block animals, performance had reached an asymptote, and there was no further improvement in rats given a third block of trials. One hour after the last trial, animals were anesthetized and prepared for recording. High-frequency stimulation induced in all groups a significant potentiation of the field EPSP measured $1 \mathrm{hr}$ after tetanization (Fig. 1B). There was an effect of training on the variance of the magnitude of potentiation $[F(4,27)=3.75, P=0.017]$, with a significant reduction of variance in both well-trained groups (Naive vs. 2 Blocks: $P<0.03$, Naive vs. 3 Blocks: $P<0.01)$. However, although the magnitude of potentiation was smallest in the 3 Blocks group, the difference between this and the Naive group was not significant $(P=0.12)$.
Table 1 shows the mean concentrations of glutamate released by veratridine, and the mean percentage differences between potentiated and nonpotentiated sides, for each of the five groups. When glutamate release between potentiated and nonpotentiated sides of the same animals was compared, the largest difference was in the Naive group (Fig. 1C; $P<0.001$ ). There was a smaller, but still significant, difference in the partially trained (1 Block) group $(P<0.01)$, but effective spatial learning completely blocked the increase in glutamate release associated with LTP in untrained or partially trained animals, with no difference between the potentiated and nonpotentiated sides in either of the well-trained groups $(P>0.4$ in both cases).

The difference between the partially trained and well-trained animals could be attributable to improved spatial learning, but it could also be the result of longer exposure to the water maze. To distinguish between these possibilities, we examined another group of rats (Visible, $n=5$ ) in a nonspatial task in the water maze. In this task the platform was visible and its location was varied from trial to trial. Rats received five blocks of six trials so that they were exposed to the water maze more often than the well-trained rats in the spatial learning task. Performance improved significantly during training (Fig. $2 \mathrm{~A} ; P<0.01$, paired $t$-test between the last trials of the first and fifth blocks). High-frequency stimulation resulted in a potentiation of the field EPSP which was similar in magnitude to the other groups (Fig. 2B). Although the induction of LTP affected glutamate release less than in naive animals, the difference between potentiated and nonpotentiated sides remained significant $(P<0.05$; Fig. $2 C)$.

\section{Discussion}

The present experiments demonstrate that spatial training results in an occlusion of the in- 
Figure 1: Spatial training is associated with an increase in glutamate release from synaptosomes and occludes the enhancement of glutamate release induced by high-frequency stimulation. (A) Spatial learning in the water maze. Mean escape latency ( \pm S.E.M.) is plotted for three groups of animals, given one (-), two (O), or three $(\square)$ blocks of six trials. There was a significant reduction in escape latency during the first block of trials $(P<0.01$ for each group, paired $t$-test between the first and last trials). A further significant improvement was achieved during the second block of trials $(P<0.005$ for each of the groups, paired $t$-test between the last trials of the first and second blocks). No further improvement was seen in the group given a third block of trials. $(B)$ Mean increase in the magnitude EPSP slope $1 \mathrm{hr}$ after high-frequency stimulation in each of trained groups and in a Naive group, which was not exposed to the water maze. (C) Well-established spatial learning abolishes the tetanus-induced enhancement of glutamate release. The graph plots the effect of training on glutamate release in synaptosomes from the potentiated side expressed as a percentage change relative to the control side. There was a signficant training effect $[F(4,22)=3.45, P<0.02$ (one-way ANOVA, applied to all five groups including the Visible group; see Fig. 2)]. Asterisks here and in Fig. 2 indicate a significant difference between the potentiated and nonpotentiated sides. $\left({ }^{*}\right) P<0.05 ;\left({ }^{* *}\right) P<0.01$. Post hoc tests indicated significant differences between trained and Naive groups: 1 Block, $P<0.04 ; 2$ Blocks, $P<0.02 ; 3$ Blocks, $P<0.01$ ).
A
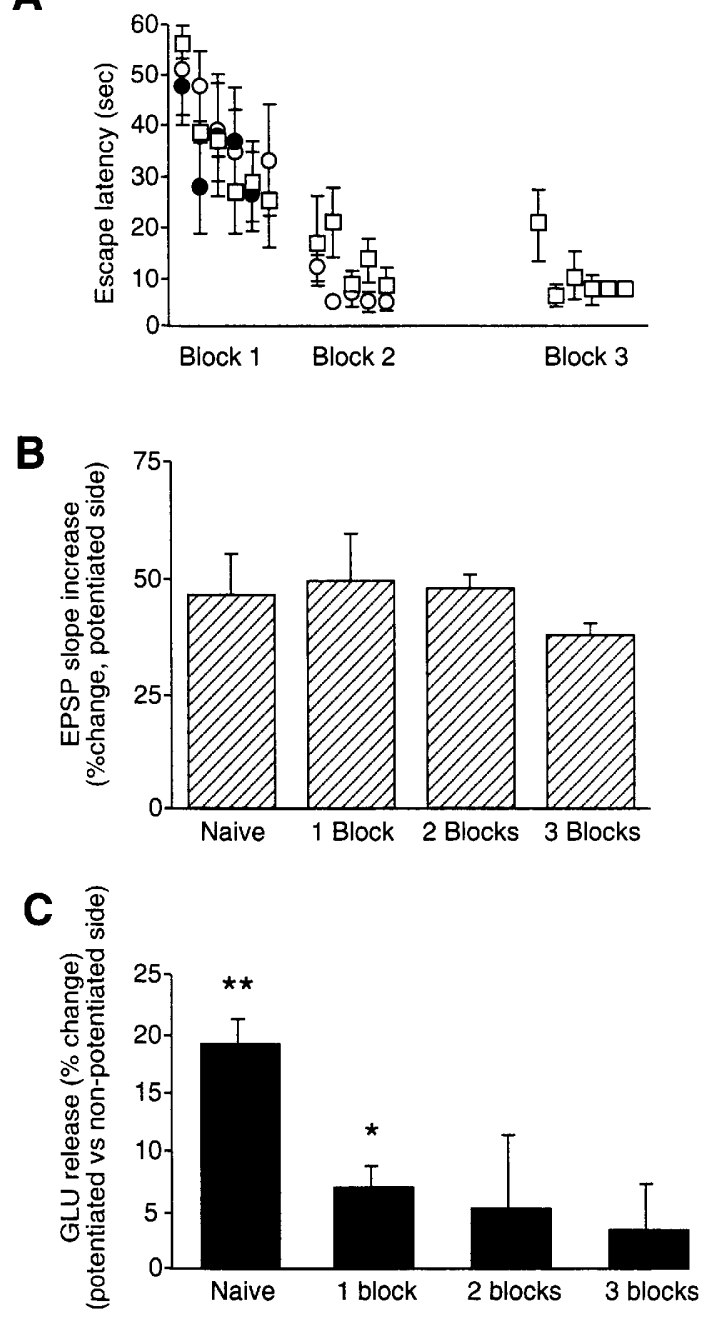

crease in glutamate release normally seen in the dentate gyrus following high-frequency stimulation of the perforant path. Our results thus provide support for earlier observations that spatial learning is accompanied by an increase in the ability of synaptosomes from the dentate gyrus to release glutamate in response to depolarization (Richter-Levin et al. 1995; McGahon et al. 1996). Partial training in the spatial task (1 Block) or training with a visible platform resulted in partial, rather than complete, occlusion of tetanus-induced enhancement of glutamate release (Figs. $1 \mathrm{C}$ and $2 \mathrm{C}$ ). This may reflect a spatial component in these two cases or, alternatively, the engagement of similar release mechanisms in nonspatial forms of learning (Laroche et al. 1987; Redini-Del Negro and Laroche 1993).

What function might such an increase in glutamate release serve? If the effect translates in vivo to a generalized increase in the excitability of gran- ule cells, increased release may represent not a specific learning mechanism but an arousal effect, enabling information to flow more readily through the dentate gyrus to areas CA3 and CA1, where, as some models assume, information is stored in autoassociative networks (McNaughton and Morris 1987; Treves and Rolls 1992). If this is the case, behavior-induced enhancements of glutamate release may be restricted to the dentate gyrus. The results of a comparison of the effects of classical conditioning on glutamate release in areas CA1, CA3, and the dentate gyrus are consistent with this view (Lynch et al. 1990).

The occlusion between tetanus-induced and training-induced changes in transmitter release suggests that similar cellular mechanisms are engaged both in LTP and in spatial training. In contrast, we were unable to detect any reduction in LTP itself after training. The experiments thus re- 
A

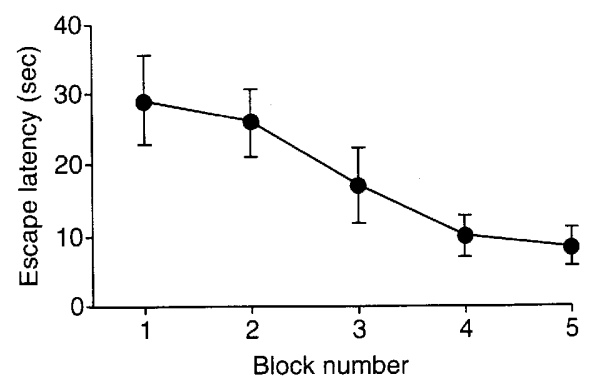

B
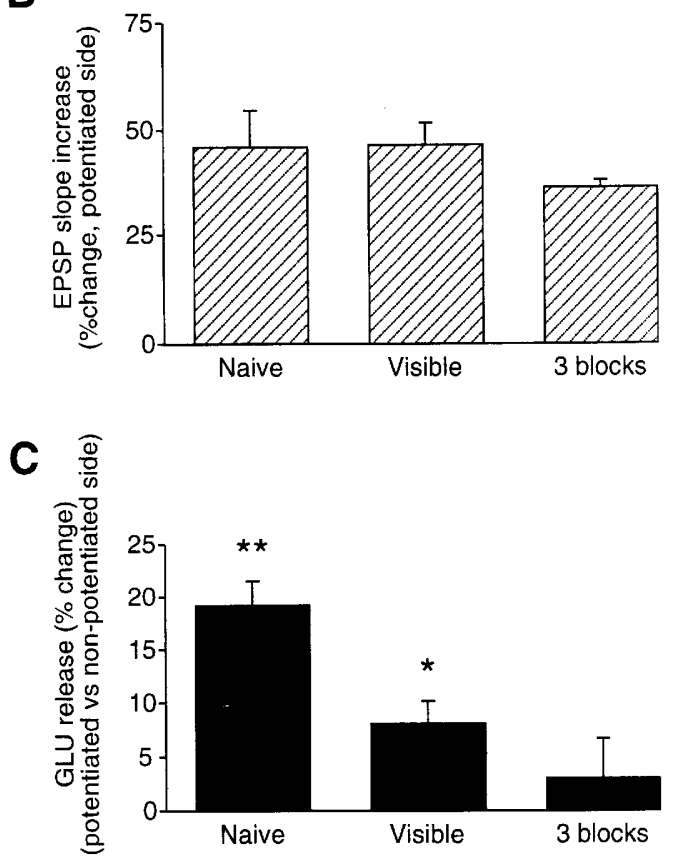

Figure 2: Training in a nonspatial water-maze task only partially occludes tetanus-induced enhancement of glutamate release. (A) Nonspatial learning in the water maze. In this task, the platform was visible, and its location changed between trials. Performance improved significantly during training $(P<0.01$, paired $t$-test comparing the last trials of the first and fifth blocks). Results are presented as averages of all trials for each block. $(B)$ The magnitude of LTP in the Visible group. Results from the Naive and 3 Blocks groups are shown for comparison. $(C)$ The effects of nonspatial training on tetanusinduced enhancement of glutamate release. The difference between sides in the Visible group was marginally smaller than in the Naive group (Visible vs. Naive, $P<0.055)$.

veal a dissociation between LTP and an increase in depolarization-induced glutamate release, an unexpected observation given the well-documented correlation between the two effects in untrained animals. For example, an increase in depolarization-induced glutamate release is seen when te- tanic stimulation leads to LTP but not when LTP is blocked by MK-801 (Canevari et al. 1994). Moreover, the two effects have a similar time course: When animals with implanted electrodes were killed 3 days after tetanus stimulation, at a time when there was substantial LTP, depolarization-induced glutamate release from dentate slices was higher on the potentiated side; 3 weeks after the tetanus stimulation, when evoked responses had declined back to baseline, there was no difference in glutamate release between control and tetanized sides (Bliss et al. 1987). A possible explanation for the apparent discrepancy between these two sets of results can be found if we suppose that in the naive animal there is availability for increased transmitter release and consequently this mechanism contributes to LTP; in contrast, in the trained animal, transmitter release at synapses activated during learning may be saturated, and postsynaptic mechanisms for expressing LTP are exploited. A similar idea has been advanced in quantal studies of LTP in area CA1; at synapses where the probability of release, $P$, is low, LTP tends to be expressed as an increase in quantal content, conventionally a presynaptic parameter; where $P$ is high to start with, LTP is expressed as an increase in quantal size, a postsynaptic parameter (Larkman et al. 1992). This line of reasoning leads to the prediction that spatial training is associated predominantly with a presynaptic form of LTP.

In conclusion, the significance of our experiments is twofold. First, the protocol that we have introduced brings the statistical power of withinanimal comparisons to bear on the question of whether learning is accompanied by the same biochemical changes as those induced by high-frequency stimulation. We have been concerned here with glutamate release in spatial learning, but the same approach can be used to ask whether any biochemical, molecular, or morphological change associated with LTP also occurs at different stages and in different types of learning. Second, our results indicate that spatial experience is associated with an increase in the efficiency with which depolarization induces glutamate release in the dentate gyrus. The function of this form of plasticity remains to be elucidated.

\section{Acknowledgments}

G.R.-L. and L.C. were supported by fellowships from Human Frontier Science Program and the European Union, respectively.

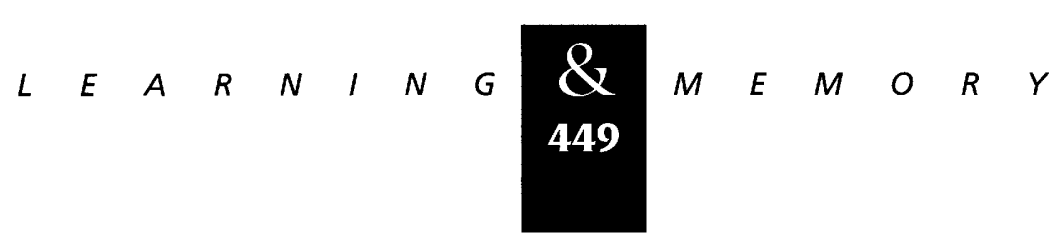




\section{Richter-Levin et al.}

The publication costs of this article were defrayed in part by payment of page charges. This article must therefore be hereby marked "advertisement" in accordance with 18 USC section 1734 solely to indicate this fact.

\section{References}

Barnes, C.A. 1995. Involvement of LTP in memory: Are we searching "under the street light"? Neuron 15: 751-754.

Bliss, T.V.P., R.M. Douglas, M.L. Errington, and M.A. Lynch. 1986. Correlation between long-term potentiation and release of endogenous amino acids from dentate gyrus of anaesthetized rats. J. Physiol. 377: 391-408.

Bliss, T.V.P., M.L. Errington, S. Laroche, and M.A. Lynch. 1987. Increase in $\mathrm{K}^{+}$-stimulated, $\mathrm{Ca}^{2+}$-dependent release of $[3 \mathrm{H}]$ glutamate from rate dentate gyrus three days after induction of long-term potentiation. Neurosci Lett. 83: $107-112$.

Canevari, L., S. Vieira, M. Aldegunde, and F. Dagani. 1992. High performance liquid chromatographic separation with electrochemical detection of amino acids focusing on neurochemical application. Anal. Biochem. 205: 137-142.

Canevari, L., G. Richter-Levin, and T.V.P. Bliss. 1994. LTP in the dentate gyrus is associated with a persistent NMDA receptor-dependent enhancement of synaptosomal glutamate release. Brain Res. 667: 115-117.

Dolphin, A.C., M.L. Errington, and T.V.P. Bliss. 1982. Long-term potentiation of the perforant path in vivo is associated with increased glutamate release. Nature 297: 496-498.

Errington, M.L., M.A. Lynch, and T.V.P. Bliss. 1987. Long-term potentiation in the dentate gyrus: Induction and increased glutamate release are blocked by D(-)aminophosphonovalerate. Neuroscience 20: 279-284.

Ghisjen, W.E.J.M., E. Besselen, V. Geukers, W. Kamphuis, and F.H. Lopes da Silva. 1992. Enhancement of endogenous release of glutamate and $\gamma$-aminobutyric acid from hippocampus CA1 slices after in vivo long-term potentiation. J. Neurochem. 59: 482-486.

Grant, S., T.J. O'Dell, K.A. Parl, P.L. Stein, P. Soriano, and E.R. Kandel. 1992. Impaired long-term potentiation, spatial learning and hippocampal development in fyn mutant mice. Science 258: 1903-1910.

Jeffery, K.J. 1997. LTP and spatial learning-Where to next? Hippocampus 7: 95-110.

Larkman, A., T. Hannay, K. Stratford, and J. Jack. 1992. Presynaptic release probability influences the locus of long-term potentiation. Nature 360: 70-73.

Laroche, S., M.L. Errington, M.A. Lynch, and T.V.P. Bliss. 1987. Increase in [3H]glutamate release from slices of dentate gyrus and hippocampus following classical conditioning in the rat. Behav. Brain Res. 25: 23-29.
Lynch, M.A. and K. Voss. 1990. Arachidonic acid increase inositol phospholipid metabolism and glutamate release in synaptosomes prepared from hippocampal tissue. J. Neurochem. 55: 215-221.

Lynch, M. A., M.L. Errington, M.P. Clements, T.V.P. Bliss, C. Redini-Del Negro, and S. Laroche. 1990. Increases in glutamate release and phosphoinositide metabolism associated with long-term potentiation and classical-conditioning. Prog. Brain Res. 83: 251-256.

McGahon, B., C. Holscher, L. McGlinchey, M.J. Rowan, and M.A. Lynch. 1996. Training in the Morris water maze occludes the synergism between ACPD and arachidonic acid on glutamate release in synaptosomes prepared from rat hippocampus. Learn. Mem. 3: 296-304.

McNaughton, B.L. and R.G.M. Morris. 1987. Hippocampal synaptic enhancement and information storage within a distributed memory system. Trends Neurosci. 10: 408-415.

McNaughton, B.L., C.A. Barnes, J. Meltzer, and R.J. Sutherland. 1989. Hippocampal granule cells are necessary for normal spatial learning but not for spatially-selective pyramidal cell discharge. Exp. Brain Res. 76: 485-496.

Morris, R.G.M. 1984. Developments of a water-maze procedure for studying spatial learning in the rat. J. Neurosci. Methods 11: 47-60.

Morris, R.G.M., E. Anderson, G.S. Lynch, and M. Baudry. 1986. Selective impairment of learning and blockade of long-term potentiation by an $\mathrm{N}$-methyl-D-aspartate receptor antagonist, AP5. Nature 319: 681-683.

Redini-Del Negro, C. and S. Laroche. 1993.

Learning-induced increase in glutamate release in the dentate gyrus is blocked by the NMDA antagonist AP5. Neurosci. Res. Comm. 13: 157-165.

Richter-Levin, G., M.L. Errington, H. Maegawa, and T.V.P. Bliss. 1994. Activation of metabotropic receptors is necessary for long-term potentiation in the dentate gyrus and for spatial learning. Neuropharmacology 33: 853-857.

Richter-Levin, G., L. Canevari, and T.V.P. Bliss. 1995. Long-term potentiation and glutamate release in the dentate gyrus: Links to spatial learning. Behav. Brain Res. 66: 37-40.

Silva, A.J., C.F. Stevens, S. Tonegawa, and Y.Y. Wang. 1992. Deficient hippocampal long-term potentiation in alpha-calcium-calmodulin kinase-II mutant mice. Science 257: 201-206.

Treves, A. and E.T. Rolls. 1992. Computational constraints suggest the need for two distinct input systems to the hippocampal CA3 network. Hippocampus 2: 189-199.

Whittaker, V.P. 1969. Handbook of neurochemistry (ed. A. Lajtha). Plenum Press, New York, NY.

Received August 10, 1995; accepted in revised form January 15, 1998.

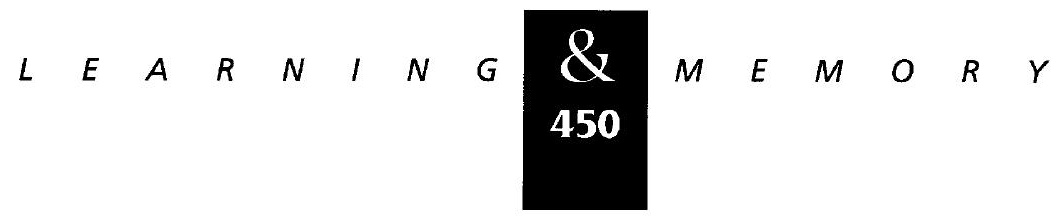




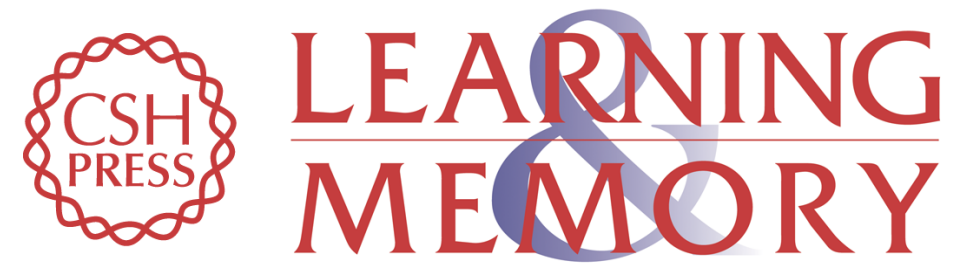

\section{Spatial training and high-frequency stimulation engage a common pathway to enhance glutamate release in the hippocampus.}

G Richter-Levin, L Canevari and T V Bliss

Learn. Mem. 1998, 4:

Access the most recent version at doi:10.1101//m.4.6.445

References This article cites 24 articles, 3 of which can be accessed free at: http://learnmem.cshlp.org/content/4/6/445.full.html\#ref-list-1

License

Email Alerting Receive free email alerts when new articles cite this article - sign up in the box at the Service top right corner of the article or click here. 